"Autonomía y sistematicidad del dispositivo represor. La Policía Federal en Neuquén (1975-1978)"

Pablo Scatizza

páginas / año 9 - n 21 Septiembre-Diciembre / ISSN 1851-992X/ pp.152-174/

2017

http://revistapaginas.unr.edu.ar/index.php/RevPaginas

\title{
Autonomía y sistematicidad del dispositivo represor. La Policía Federal en Neuquén (1975-1978) ${ }^{1}$
}

\section{Autonomy and systematicity of the repressor device. The Federal Police in Neuquén (1975-1978)}

\author{
Pablo Scatizza \\ Facultad de Humanidades, \\ Universidad Nacional del Comahue, Argentina \\ pscatizza@gmail.com
}

\begin{abstract}
Resumen
En este artículo se expone la dinámica represiva propia que fue adquiriendo la Policía Federal en Neuquén (Argentina) en los años previos y durante la última dictadura militar. En este sentido, se destaca cómo se fue articulando su accionar con las otras fuerzas e instituciones con las cuales constituyó el dispositivo represor que caracterizó ese período, cuáles fueron algunas características del universo moral de sus miembros y la manera en que el mismo se dinamizó con la "lucha antisubversiva", y cuál fue el grado de autonomía que tuvo respecto de las Fuerzas Armadas al momento de actuar. El objetivo de este recorrido es, en definitiva, realizar un aporte más a los estudios sobre represión y violencia política que se vienen desarrollando hace algunos años en diversas partes del país, con la idea de armar un mapa cada vez más completo sobre la manera en que se desplegó el plan represivo que caracterizó a las décadas del sesenta y setenta en el cono sur.
\end{abstract}

Palabras clave: Dictadura; Represión; Policía Federal; Neuquén; Norpatagonia

\footnotetext{
Abstract

This article analyses the repressive dynamic that the Federal Police in Neuquén (Argentina) acquired in the previous years and during the last military dictatorship. It is highlighted how

${ }^{1}$ Un borrador de este trabajo fue presentado como ponencia -y no publicado en Actas- en las XVII Jornadas Departamentos/Interescuelas de Historia, Mar del Plata, 9-11 de agosto de 2017. Agradezco especialmente a quienes evaluaron este artículo para su publicación aquí -cuyo nombre desconozcopor sus oportunas y precisas observaciones.

Esta obra está sujeta a la Licencia Reconocimiento-NoComercial-CompartirIgual 4.0 Internacional de Creative Commons. http://creativecommons.org/licenses/by-nc-sa/4.0/

(cc) EY-NC-SA
} 
its action was articulated with the other forces and institutions with which this police constituted the repressive device that characterized that period, what were some characteristics of the moral universe of its members and the way it was part of the "antisubversive struggle", and what was the degree of autonomy that it had with respect to the Armed Forces at the moment of acting. The aim of this reflection is, in short, to make a contribution to the studies on repression and political violence that have been developing since some years ago in different regions of this country, with the idea of putting together an increasingly complete map on the way that the repressive plan that characterized the decades of the sixties and seventies in the southern cone was deployed.

Keywords: Dictatorship; Repression; Federal Police; Neuquén; North Patagonia

\section{Juego de escalas}

Mirar un cuadro. Por ejemplo, El palacio papal, Avignon, del puntillista Paul Signac (c. 1900). Observado a cierta distancia, la forma del palacio queda separada claramente de un cielo que funde azules, morados y verdes. La luz que refleja el agua que lo rodea permite distinguir la orilla arbolada que continúa al puente de arco inconcluso y, el conjunto, es fácilmente apreciable. Pero al acercarnos al lienzo, la imagen comienza a desdibujarse para darle paso a un conglomerado de puntos de diversos tonos de colores cuyos bordes no son distinguibles a la distancia. Minúsculas pinceladas que ya no parecen puntos siquiera, sino trazos cuyas líneas e irregularidades devela la cercanía de la mirada. Cada color toma matices no percibidos a la distancia, y por momentos se torna difícil diferenciar dónde comienza uno y dónde termina el otro.

Pero lo interesante no termina allí, sino cuando volvemos a alejarnos para apreciar nuevamente el cuadro en su conjunto. ¿Qué sucedió? ¿Por qué lo que vemos ya no es lo mismo, si el cuadro es el mismo? ¿Por qué perdió simplicidad, si es que alguna vez la tuvo?

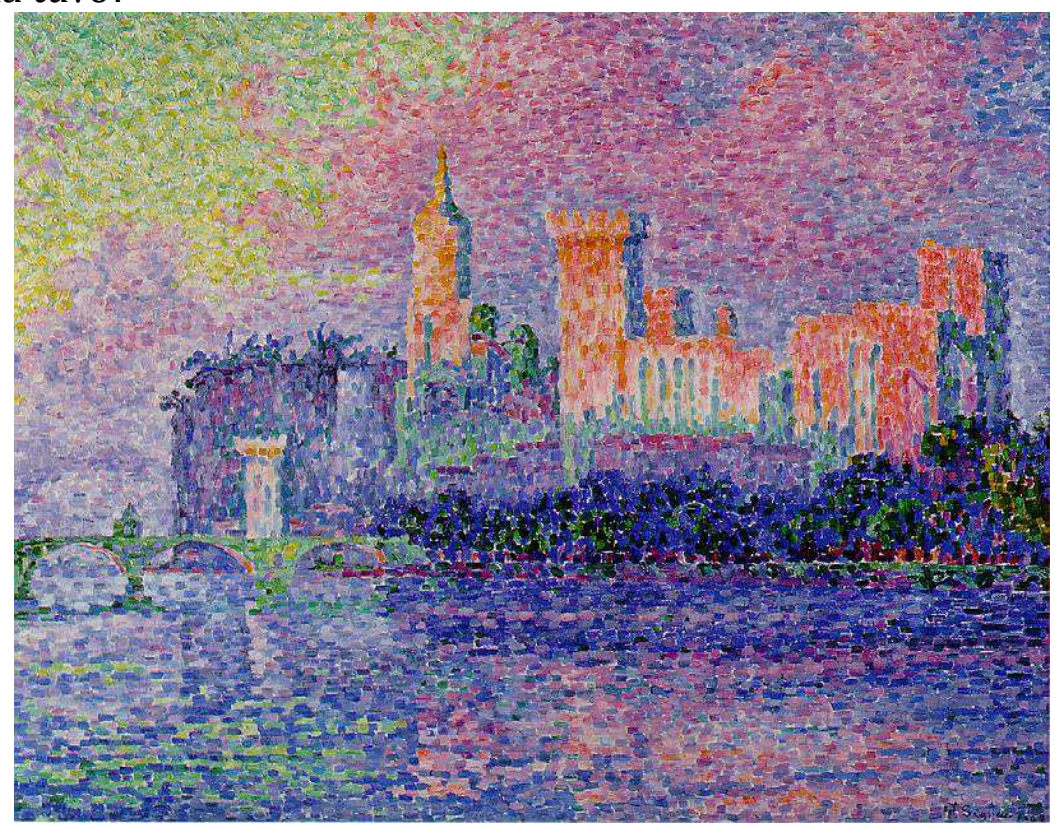




\section{“Autonomía y sistematicidad del dispositivo represor. La Policía Federal en Neuquén (1975-1978)"}

Algo similar suele ocurrir en el oficio de historiar, cuando cambiamos la lente para observar y estudiar esos objetos de investigación que hemos construido con la pretensión de conocer un pasado que no deja de reconfigurarse. ¿En qué medida el acto de modificar la escala de análisis y de nuestros niveles de observación, para ahondar en lo que a simple vista podrían ser matices, nos puede permitir alcanzar una mayor comprensión de los procesos históricos? ¿Qué sucede cuando nos detenemos en aquello que a la distancia parecía un dato complementario o incluso menor, y vemos que más de cerca conformaba en sí mismo un problema, y cuyo análisis complejiza -y en cierta medida vuelve más profundo- el conocimiento que ya teníamos? Sería como detenernos en la pincelada más pequeña, aquella que a la distancia puede pasar desapercibida o ser parte de un nimio detalle, como "punto de partida de un movimiento más amplio hacia la generalización". ${ }^{2}$ En especial cuando luego de sumergirnos en esos "detalles" -que generalmente no son tales- nos alejamos del cuadro que estamos analizando y volvemos a mirar la obra en su conjunto. Un movimiento de lo micro a lo macro que produce "efectos de conocimiento y que puede ser puesta al servicio de estrategias de conocimiento" para decirlo con las propias palabras de Jacques Revel- donde lo que cuenta, justamente, no es la elección de la escala en particular sino el "principio de variación".3

Debido a las características propias de nuestro objeto de investigación (que hemos comenzado a construir hace ya casi una década e involucra a la represión estatal, sus lógicas y modalidades, cuyo primer acercamiento lo circunscribe a las décadas de 1960/1970 y por lo cual forma parte de un proceso histórico que posee múltiples aristas que se desarrolló en diferentes espacios regionales adquiriendo sus propias especificidades) pensar en una variación de la escala de observación nos remite necesariamente a dos planos yuxtapuestos. Uno espacial, que nos permite (o impulsa a) estudiar a este objeto en términos regionales/locales en tensión con "lo nacional" o metropolitano, y otro que nos lleva a (o exige) aumentar o disminuir el tamaño de las diferentes unidades de análisis desde las cuales pretendamos abordarlo, modificando con ello la forma y la trama que de lo que pretendemos conocer. En este sentido, nuestro trabajo intenta jugar dentro de este doble plano analítico, buscando reconstruir con mayor precisión el entramado del dispositivo

\footnotetext{
2 Giovanni Levi, se, "Sobre microhistoria", en Peter Burke (ed.). Formas de hacer historia. Madrid, Alianza, 1994, p. 124. Sin dudas esta reflexión -tanto como la perspectiva metodológica sobre la que se han apoyado nuestras investigaciones sobre la dinámica represiva en Norpatagonia- sigue la huella de los microhistoriadores italianos, en especial el recién citado Giovanni Levi y Carlo Ginzburg, además de las contribuciones teóricas sobre los cambios de escalas de observación de Jacques Revel en Juego de escalas. Experiencias de micronalálisis. Buenos Aires, UNSAM Edita, 2015 [1996] (obra que dirige y en la que autores como Bernard Lepetit, Simona Cerutti, Edoardo Grendi y el propio Giovanni Levi, entre otros, desarrollan los alcances, problemas y potencialidades de esta perspectiva teórico-metodológica).

3 “(...) jugar con las escalas de representación en cartografía no equivale a representar una realidad constante en dimensiones más grandes o más pequeñas, sino a transformar el contenido de la representación -es decir, la elección de lo que es representable. Observemos que la dimensión "micro" no goza al respecto de ningún privilegio en particular. Lo que cuenta es el principio de variación, no la elección de una escala en particular" (Jacques Revel, Juego de escalas...0p. Cit. p. 24)
} 
que hizo posible el despliegue represivo en la Patagonia Norte durante los años setenta. Si ya la propia escala de observación desde la cual abordamos en una primera instancia este objeto era considerablemente menor a la utilizada en otros estudios similares ${ }^{4}$-no solo por la "regionalización" del objeto sino por el nivel de detalle que fue posible observar al aumentar la lente-, ahora esta escala se reduce un poco más en busca de niveles más profundos de lo real, intentando complejizar su conocimiento. ${ }^{5}$

Para decirlo en términos metodológicos más concretos, el problema que aquí nos interesa trabajar es la tensión entre la sistematicidad que caracterizó al dispositivo represor durante la última dictadura militar y sus años previos, y los diferentes niveles de autonomía que adquirieron determinadas agencias represivas que lo constituyeron. Para ello, hemos aumentado aún más la lente para enfocarnos con mayor precisión en la Delegación Neuquén de la Policía Federal Argentina (PFA), una unidad de análisis que si bien ya hemos abordado parcialmente en otros trabajos $^{6}$, ahora lo haremos a partir de ciertos interrogantes específicos sobre los cuales aún no habíamos trabajado. Más concretamente, con la intención de establecer la dinámica propia que fue adquiriendo esta fuerza policial como parte del dispositivo represor desde los años previos al golpe de Estado; cómo se fue articulando con las otras fuerzas e instituciones que lo conformaron, cuáles eran algunas características del universo moral de sus miembros y cómo el mismo se dinamizó con la "lucha antisubversiva"; y cuál fue el grado de autonomía que tuvo respecto de las Fuerzas Armadas al momento de actuar.

Cuando hablamos de autonomía, nos referimos puntualmente al grado o niveles de independencia que tuvieron las distintas fuerzas policiales y de seguridad en la planificación y ejecución de las acciones represivas ${ }^{7}$, respecto de las Fuerzas Armadas bajo cuyo control operacional estuvo el accionar "antisubversivo" desde

\footnotetext{
${ }^{4}$ Nos referimos a trabajos que hoy sin duda son referenciales en relación con los estudios sobre represión, violencia política y dictadura, como los de Eduardo Luis Duhalde. El Estado Terrorista Argentino. Quince años después. Buenos Aires, Eudeba, 1999; Marcos Novaro y Vicente Palermo. La dictadura militar (1976/1983). Del golpe de estado a la restauración democrática. Buenos Aires, Paidós, 2006; Prudencio García. El drama de la autonomía militar. Alianza, Madrid, 1995; Pilar Calveiro. Poder y desaparición. Los campos de concentración en Argentina. Buenos Aires, Colihue, 2006, entre muchos otros más.

${ }^{5}$ Subyace en esto que decimos las palabras de Lepetit al afirmar: "Así pues, la multiplicación controlada de las escalas de observación puede producir mayores conocimientos si se postula la complejidad de lo real -los principios de la dinámica social son plurales y se leen según configuraciones causales diferentes- y su inaccesibilidad -la palabra final nunca está dada y la modelización siempre debe recomenzarse" (Bernard Lepetit. "De la escala en la historia". En Jacques Revel. Juego de escalas... Op. Cit

6 Ver Pablo Scatizza. "Centros clandestinos de detención en el Comahue. Una reflexión conceptual". Contenciosa, Año I, Nro. 2, primer semestre 2014 [En línea en http://www.contenciosa.org/Sitio/VerArticulo.aspx?i=18; Un Comahue violento. Dictadura, represión y juicios en la Norpatagonia argentina. Buenos Aires, Prometeo, 2016; "Represión 'antisubversiva' en la Norpatagonia. Estrategias estatales y paraestatales de persecución política en Neuquén y Río Negro (1973-1976)". Papeles de Trabajo, 10 (17), pp. 52-72

${ }^{7}$ Subrayamos esto para descartar otros posibles grados de autonomía que podría haber adquirido (o no), como autonomía económica, autonomía institucional, autonomía doctrinal u otras formas de autarquía. Ver Prudencio García. El drama de la autonomía militar. Op. cit., pp.350-352
} 


\section{"Autonomía y sistematicidad del dispositivo represor. La Policía Federal en Neuquén (1975-1978)"}

octubre de $1975 . .^{8}$ Ciertos grados de "libertad" que adquirieron, aun formando parte de un entramado represivo que actuó de manera sistemática de acuerdo a una planificación previamente desarrollada. Cabe señalar que esta búsqueda es tributaria en cierta medida de algunos de los trabajos que ha realizado Gabriela Águila, destacando la manera en que se dio esta tensión en la ciudad de Rosario y alrededores. ${ }^{9}$ Asimismo, esta modificación en la escala de observación se viene utilizando metodológicamente en torno al tema de la represión y la violencia política hace ya algunos años y en diversas partes del país, con la idea de armar un mapa cada vez más complejo sobre la manera en que se desplegó el plan de exterminio que caracterizó a las décadas del sesenta y setenta en el cono sur. ${ }^{10}$

Un elemento motivador para avanzar en esta búsqueda fue la dificultad de poder ensayar hipótesis lo suficientemente sólidas que pudieran explicar ciertos procedimientos realizados por la Policía Federal durante la última dictadura y sus años previos, y que a primera vista escapaban a la lógica de funcionamiento del dispositivo represor. Por un lado, acciones que a la distancia parecían no estar vinculadas a la "lucha contra la subversión", pero que al mirar más detenidamente quedaba en evidencia que sí lo estaban. Y por otro, el despliegue de cierto grado de autonomía en la planificación y ejecución de acciones respecto del Ejército, mucho mayor que el que tuvieron el resto de las fuerzas en la Norpatagonia. Indicios que dejaban en evidencia que no se trató de sólo una institución represiva más dentro de este entramado, y que era necesario reformular algunas preguntas para poder seguir avanzando. Si bien no resulta novedoso el protagonismo indiscutible que tuvo

\footnotetext{
${ }^{8}$ El 6 de octubre de 1975 fueron sancionados los decretos 2770, 2771 y 2772, por los cuales se delegaba en las Fuerzas Armadas la intervención directa en materia de seguridad interna con el objetivo concreto y explícito de aniquilar a la "subversión".

9 Ver por ejemplo: Gabriela Águila. "La represión en la historia reciente argentina: fases, dispositivos y dinámicas regionales". En Gabriela Águila y Luciano Alonso (coord.). Procesos represivos y actitudes sociales. Entre la España franquista y las dictaduras del Cono Sur. Buenos Aires, Prometeo, 2013, pp.97-121, y Dictadura, represión y sociedad en Rosario... Op.Cit

${ }^{10} \mathrm{Gran}$ parte de estos estudios se desarrollan en el marco de la Red de Estudios sobre Represión y Violencia política (RER), los cuales están siendo plasmados en jornadas de trabajo y diversas publicaciones en libros y revistas académicas. Ver Gabriela Águila, Santiago Garaño y Pablo Scatizza (coords.). Represión estatal y violencia paraestatal en la historia reciente argentina: nuevos abordajes a 40 años del golpe de Estado. La Plata, Ediciones FaHCE, Universidad Nacional de La Plata, 2016. Disponible en http://www.memoria.fahce.unlp.edu.ar/libros/pm.423/pm.423.pdf. A pesar de que la preocupación en torno a la tensión entre miradas "nacionales" y "regionales/locales" -y que sólo en cierta medida se relacionan con el "juego de escalas" al que nos estamos refiriendo- no es nuevo dentro del campo de la Historia Reciente (ver por ejemplo, Gabriela Águila, "La dictadura militar argentina: interpretaciones, problemas y debates". Páginas. Revista digital de la Escuela de Historia. Rosario, UNR, año 1, $\mathrm{n}^{\circ} 1$; y de la misma autora, "La represión en la historia reciente argentina: perspectivas de abordaje, conceptualizaciones y matrices explicativas". Contenciosa. Revista sobre violencia política, represiones y resistencias en la historia iberoamericana, Santa Fe, UNL, 2013, pp. 113), sí lo es de alguna manera la reflexión teórico metodológica sobre esta perspectiva microanalítica, como quedó plasmado en un dossier dedicado exclusivamente a ello publicado en 2015 (ver dossier "Dossier: Las escalas de análisis en los estudios sobre el pasado reciente". Avances del Cesor, Año XII, $\mathrm{N}^{\circ} 12$, primer semestre de 2015, disponible en http://web2.rosarioconicet.gov.ar/ojs/index.php/AvancesCesor/issue/view/44/showToc)
} 


\section{Pablo Scatizza}

la Policía Federal como institución en el desarrollo de la represión ${ }^{11}$, creemos que la posibilidad de cambiar la escala espacial y profundizar el análisis de las características que adoptó esta fuerza por fuera de los grandes centros urbanos puede brindar más elementos para comprender mejor su accionar global. Especialmente, si tenemos en cuenta que más allá de haber actuado bajo control operacional de las Fuerzas Armadas luego de sancionados los "decretos de aniquilamiento" de octubre de 1975, su propio y particular despliegue en todo el territorio nacional bajo una coordinación centralizada en la Capital Federal hizo de su participación un elemento clave del dispositivo represor.

Una breve aclaración conceptual: cuando hablamos de dispositivo represor no pensamos la nación de "dispositivo" en tanto artefacto, mecanismo o conjunto de piezas que cumple una función determinada, sino en los términos de Michel Foucault: como un heterogéneo conjunto de discursos, instituciones, instalaciones arquitectónicas, decisiones reglamentarias, leyes, medidas administrativas, enunciados científicos, proposiciones filosóficas y morales; una red de prácticas discursivas y no discursivas; "una especie -digamos- de formación que, en un momento histórico dado, tuvo como función mayor la de responder a una urgencia". ${ }^{12}$ ¿En qué medida cambia nuestro entendimiento sobre la represión el pensarla tras esta matriz conceptual? Es cierto que no lo hace radicalmente, pero sí nos exige, de alguna manera, a tener presente que el desarrollo del proyecto represivo implicó mucho más que la suma de un conjunto de instituciones cuya naturaleza era ya, de por sí, represiva, sino que en cierta medida configuró un entramado; una red cuyos hilos fueron esas instituciones -para lo cual hubieron de reconfigurar parte de sus prácticas y saberes-, pero también discursos y significaciones, toda una red de relaciones que, más que habilitarlo y dejarlo actuar, formó parte de su actuación.

El corpus documental en el cual se sustenta nuestro análisis es en gran medida de origen judicial, vinculado a la causa "Reinhold, Oscar Lorenzo y otros s/ Delitos c/ la libertad y otros" Expte. № 8736/2005 Juzgado Federal № 2 de Neuquén, Secretaría № 2, correspondiente al juicio por delitos de lesa humanidad que comenzó a desarrollarse en Neuquén en 2005. Como parte de esa causa ya se ventilaron públicamente cinco tramos (la causa fue fragmentada y dividida en tramos, según los cuales se fueron realizando los sucesivos debates orales ${ }^{13}$ ), por lo

\footnotetext{
${ }^{11}$ Entre otros, ver Eduardo Luis Duhalde. El Estado Terrorista Argentino. Quince años después. ob. cit.; Martín Andersen. La policía. Pasado, presente y futuro. Buenos Aires, Sudamericana, 2002; Marcos Novaro y Vicente Palermo. La dictadura militar (1976/1983)... ob. cit.; Marcelo Larraqui. Lopez Rega. El peronismo y la Triple A. Buenos Aires, Punto de Lectura, 2007; Gabriela Águila. Dictadura, represión y sociedad en Rosario, 1976/1983. Un estudio sobre la represión y los comportamientos y actitudes sociales en dictadura. Buenos Aires, Prometeo, 2008; Ariel Eidelman. El desarrollo de los aparatos represivos del Estado argentino durante la "Revolución Argentina", 1966-1973, Tesis de Doctorado, UBA, 2010. Disponible en http://repositorio.filo.uba.ar/handle/filodigital/1412

${ }^{12}$ Michel Foucault. "El juego de Michel Foucault". En Saber y Verdad. Madrid, Las Ediciones de la Piqueta, 1984, p. 124

${ }^{13}$ Para más información sobre el desarrollo de esta causa judicial, ver Pablo Scatizza. Un Comahue violento... Op. Cit., pp. 245-269
} 


\section{"Autonomía y sistematicidad del dispositivo represor. La Policía Federal en Neuquén (1975-1978)"}

cual gran parte de la documentación se encuentra disponible, aunque no públicamente accesible. ${ }^{14}$ En este sentido, la asistencia a los debates orales ha permitido registrar numerosos testimonios $\mathrm{y}$ presenciado las numerosas exposiciones probatorias. Asimismo, se han utilizado diarios de la época y realizado entrevistas personales. Por último, y como hemos referido más arriba, cabe señalar que no es la primera vez que analizamos el funcionamiento de la PFA, pero sí de la manera en que sigue a continuación, tratando de acercar aún más la lente para precisar mejor su papel como parte del dispositivo. En este sentido, y en el intento de evitar repeticiones con lo expuesto en otros trabajos, haremos referencia a ellos cuando sea necesario.

\section{La fuerza se prepara}

Como hemos demostrado en otros trabajos ${ }^{15}$, a comienzos de 1974 se evidencia en la Subzona $52^{16}$ una escalada de violencia hacia las militancias y todo tipo de expresiones del amplio arco de la izquierda que bien puede pensarse como el sustrato sobre el cual germinó el dispositivo represor que luego caracterizó a la última dictadura. Una serie de actos intimidatorios (atentados con bombas, tiroteos, amenazas de muerte) que algunos analistas del momento ya le adjudicaban a fuerzas peronistas de derecha y ligadas al ministro de Bienestar Social, José López Rega, que actuarían en sintonía fina con el proceso de "depuración interna del peronismo" iniciado oficialmente ${ }^{17}$ en octubre de 1973, con la firma del "documento reservado" por parte de Perón que promovía la lucha contra la "infiltración marxista” dentro del movimiento peronista "a través de todos los medios que se consideraran eficientes".18 Cabe recordar que en sincronía con esto último, y como parte de la decisión del gobierno y su entorno de incrementar la represión hacia los sectores de izquierda y contestatarios, el presidente interno Raúl Lastiri reincorporó por

\footnotetext{
14 Lamentablemente no hay ningún repositorio o centro documental donde los investigadores/as podamos acceder libremente a consultar el material. Para acceder a él, es necesario buscar por los intersticios que deja el sistema, como abogados/as querellantes, testigos de la causa, contactos internos en el Poder Judicial, etc.

15 Cfr. Pablo Scatizza. Un Comahue violento. Dictadura, represión y juicios en la Norpatagonia argentina. Buenos Aires, Prometeo, 2016; Pablo Scatizza. “Represión 'antisubversiva' en la Norpatagonia. Estrategias estatales y paraestatales de persecución política en Neuquén y Río Negro (1973-1976)”. Papeles de Trabajo, 10 (17), pp. 52-72

${ }^{16}$ Una de las características fundamentales que adoptó y permitió el desarrollo del plan de exterminio llevado a cabo por las FFAA fue la división del territorio nacional en cinco "zonas de seguridad", cada una de ellas a su vez con "subzonas", "áreas" y "subáreas". Para el la región aquí analizada, la Subzona 52 abarcó a la totalidad de la provincia de Neuquén, y más de la mitad de la provincia de Río Negro (Pablo Scatizza. Un Comahue violento....0p. Cit.)

17“Oficialmente", dado el estado público que tomó el documento, pero que ya había mostrado su antecedente varios meses antes en la propia Masacre de Ezeiza y en el accionar de grupos paraestatales como la Triple A y la Concentración Nacional Universitaria.

${ }_{18}^{18}$ Marina Franco. Un enemigo para la nación. Orden interno, violencia y "subversión”, 1973-1976. Buenos Aires, FCE, 2012; Hernán Merele, "La "depuración ideológica" del peronismo en el Partido de General Sarmiento (Buenos Aires). Una aproximación a partir del caso de Antonio "Tito" Deleroni", Ponencia presentada en las Primeras Jornadas de Historia Reciente del Conurbano Bonaerense Norte y Noroeste, UNGS, Buenos Aires, agosto de 2013.
} 
decreto el 11 de octubre de 1973 -un día antes de que Perón asumiera su tercer presidencia- al comisario Juan Ramón Morales y al subinspector Rodolfo Eduardo Almirón. ${ }^{19}$ Ambos al servicio activo de la Policía Federal como miembros de la custodia del General Perón y del propio López Rega, devenidos luego en dos de los máximos responsables de la Triple $\mathrm{A}$, cuyo primer acto público reconocido, concretado pocas semanas después, fue el atentado contra el senador radical Hipólito Solari Yrigoyen. ${ }^{20}$ También es preciso tener en cuenta que, ya instalado en la Casa Rosada, Perón impulsaría rápidamente cambios sustanciales hacia el interior de la PFA, con el reingreso por decreto a esa fuerza de Alberto Villar como subjefe y de Luis Margaride como Superintendente de Seguridad Federal -el 29 de enero de 1974- a partir de lo cual se concretaría la articulación triangular entre la Policía Federal, el Ministerio de Bienestar Social y la Triple A. ${ }^{21}$

En lo que respecta a Neuquén, la Delegación de la PFA en esta capital comenzaría a integrarse a la dinámica represiva recién a comienzos de 1975, con la llegada a esta dependencia de actores clave de su accionar hasta entrado el gobierno de la dictadura. Personajes que, según consta en sus legajos, tenían abultada experiencia en tareas de Inteligencia así como relación estrecha con las Fuerzas Armadas. El primero de ellos fue el comisario Jorge Ramón González, quien asumió la jefatura de la Delegación el 8 de enero de 1975, y como tal sería el principal responsable del accionar represivo de esa fuerza. ${ }^{22}$ Desde entonces, y en el transcurso de ese año, una veintena de agentes de distinto rango llegaron a Neuquén, muchos de ellos con acreditada participación en el desarrollo de la represión, como el subcomisario Jorge Alberto Soza, y los agentes Miguel Ángel Cancrini, Ricardo Arean, Javier Fernández y Gustavo Alberto Sommer.23

Coincidentemente con el armado de la PFA en esta ciudad haría su desembarco en la Universidad Nacional del Comahue (UNCo) la "misión Ivanisevich", bajo la conducción del fascista rumano Dionisio Remus Tetu. ${ }^{24}$ Durante la gestión de

\footnotetext{
${ }^{19}$ Ambos continuarían junto a López Rega hasta su renuncia al Ministerio de Bienestar Social y su partida a España en julio de 1975, cercado por las denuncias penales en su contra, huyendo luego con él a ese país, también como custodios del ahora flamante embajador extraordinario y plenipotenciario (Marcelo Larraquy. López Rega, el peronismo y la Triple A. Op. Cit., pp. 345-348) ${ }^{20}$ Sobre la Triple A y su accionar, ver Horacio Paino. Historia de la Triple A. Montevideo, Editorial Platense, 1984; Ignacio González Janzen. La Triple A. Buenos Aires, Contrapunto, 1987; Martín Andersen. Dossier Secreto. El mito de la guerra sucia. Buenos Aires, Planeta, 1993; Prudencio García. El drama de la autonomía militar. Argentina bajo las juntas militares. Madrid, Alianza, 1995; Sergio Buffano. "Perón y la Triple A". Lucha Armada en la Argentina Año 1 - N³3, Buenos Aires, 2005; Marcelo Larraquy. López Rega, el peronismo y la Triple A... Op. Cit.

${ }^{21}$ El mismo día que asumen Villar y Margaride la Triple A hizo público un comunicado con una "lista negra" de personalidades que debían "ser ejecutadas inmediatamente donde se las encuentre" (Sergio Buffano, "Perón y la Triple A". Op. Cit. p.26.

${ }^{22} \mathrm{La}$ información sobre el personal de la PFA se encuentra en el Requerimiento Fiscal de Elevación a Juicio de junio de 2011, en la causa "Reinhold, Oscar Lorenzo y otros s/ Delitos c/ la libertad y otros" Expte. № 8736/2005 Juzgado Federal № 2 de Neuquén, Secretaría № 2. (En adelante, RFEJ 2011 para referir al Requerimiento, y Causa Reinhold para referir al expediente judicial)

${ }^{23}$ RFEJ 2011

${ }^{24}$ La llegada de Remus Tetu a esta universidad respondió a la decisión emanada desde el Ministerio de Educación de la Nación que desde agosto de 1973 había quedado en manos de Oscar Ivanisevich, cuya misión "era justamente retomar el control de las universidades en una orientación acorde a la
} 


\section{"Autonomía y sistematicidad del dispositivo represor. La Policía Federal en Neuquén (1975-1978)"}

poco más de 11 meses al frente del rectorado, Tetu se propuso "depurar la estructura curricular de la UNC (sic) de todo lo tendencioso, unilateral y partidista (...) así como de todo lo que pueda atentar contra la seguridad del país (...)", para lo cual resolvía "dar por terminadas todas las llamadas experiencias o innovaciones pedagógicas y didácticas en el dictado de clases", y advertía que "toda transgresión a las presentes disposiciones que pudieran constatarse serán sancionadas de inmediato con el máximo rigor y sin contemplación alguna". 25 Se lo propuso y lo hizo. No sólo expulsó a más de 130 trabajadores/as docentes y no docentes de esta universidad, sino que incorporó un grupo de choque que protagonizarían no pocas acciones represivas. ${ }^{26}$ Entre ellas, el atentado a la agencia neuquina del diario Río Negro y a una reconocida librería de esta ciudad (la primera mediante el tiroteo de las oficinas y la segunda a través de un artefacto explosivo), así como la persecución y hostigamiento a estudiantes y trabajadores de la universidad. ${ }^{27}$

Entre los personajes que ingresarían a formar parte del "personal de custodia" de Remus Tetu se encontraba Raúl Guglielminetti, un engranaje fundamental dentro de la maquinaria de persecución y exterminio, y cuyo desempeño permite, de alguna manera, demostrar el vínculo estrecho e ineludible entre lo estatal y lo paraestatal del dispositivo represor, antes y durante la última dictadura. Y esto, debido a la multiplicidad de roles que cumplió durante los años

dirección política del gobierno (nacional)" (Juan Quintar, Beatriz Gentile, Susana Debattista y Carla Bertello. "La Universidad Nacional del Comahue en los años 70: de la rebelión creativa a la reacción autoritaria”. En AA.VV., Universidad Nacional del Comahue 1972-1997. Una historia de 25 años. Neuquén, Educo, 1998, p. 59). Una dirección política que estaba delineada por los sectores ortodoxos de la derecha peronista que habían quedado en torno a Isabel luego de la muerte de Perón, en claro enfrentamiento con las distintas expresiones de izquierda que operaban en el país. Remus Tetu, un exiliado rumano que había integrado el movimiento fascista, ultranacionalista y antisemita Guardia de Hierro, asumió como rector de la UNCo el 4 de enero de 1975, y permaneció al frente de esta casa de estudios hasta el 19 de noviembre de ese mismo año. En todo ese período, Tetu también fue rector interventor de la Universidad Nacional del Sur, en Bahía Blanca, donde puso en práctica una serie de acciones represivas y persecutorias muy similares a las que impondría en Neuquén (sobre el accionar de Tetu en Bahía Blanca, ver Ana Belén Zapata. "Andamios de experiencias". Conflictividad obrera, vigilancia y represión en Argentina. Bahía Blanca, 1966-1976. Tesis doctoral, Universidad Nacional de la Plata, 2014. Mimeo. Para más información sobre Remus Tetu, ver Diego Kenis. “¿Quién fue Remus Tetu?", Agencia Paco Urondo, 3/10/2015. Disponible en http://www.agenciapacourondo.com.ar/ddhh/quien-fue-remus-tetu. Sobre su actuación en la Universidad Nacional del Comahue, ver Humberto Zambón. La misión Tetu en el Comahue. Neuquén, Educo, 2008).

${ }^{25}$ Resolución UNC Nº 0056 del 6/2/75

${ }^{26}$ Este grupo de choque estuvo conformado por nueve personas contratadas por Remus Tetu, con el argumento de la "necesidad de contar con custodia de los edificios de esta Universidad" (Resolución "I" 0411 del 14 de abril de 1975). Habría sido mediante estos hombres que el ex Guardia de Hierro ejecutaría las acciones represivas que se le adjudican a la Triple A. Si bien hay una memoria consensuada que señala a Tetu como el cerebro ejecutor de esa fuerza paraestatal -incluso un ex comisario asegura que en una reunión de la comunidad informativa el propio Remus Tetu informó que se iban "a incorporar a la vida neuquina nueve hombres que pertenecían a la Triple A" (Testimonio de Antonio Casal en Tribunal Oral en los Criminal Federal, "Causa Luera”, 7/08/2012)hay algunos cabos sueltos que nos generan dudas respecto a la supuesta organicidad que pudieron haber tenido a este "somatén criollo". Nos extendemos más sobre esto en Un Comahue violento...op. cit. pp. 55-68.

27 Para más detalle, ver Scatizza, Pablo. ““Represión 'antisubversiva' en la Norpatagonia...” op. cit. 


\section{Pablo Scatizza}

que estuvo en esta región, mientras revistaba en el Ejército Argentino como Personal Civil de Inteligencia (PCI), desempeñando funciones en el Destacamento de Inteligencia 182 de Neuquén desde fines de diciembre de 1970, hasta mayo de 1976. ${ }^{28}$ Reconocido en todo el país por su pertenencia al Batallón 601 de Inteligencia y por haber participado en delitos de lesa humanidad en varias partes de Argentina y América Latina, Raúl Guglielminetti (o "mayor Guastavino", como se hacía llamar) se ha transformado en uno de los íconos del plan sistemático de represión en la región. Llegó a la Neuquén durante las huelgas de El Chocón hacia fines de 1969, se convirtió en un personaje de muy alto perfil y muy reconocido en el ambiente político, universitario y de los medios de comunicación. Durante el período en que estuvo bajo la órbita del Ejército, Guglielminetti "trabajó" además como periodista en la principal radio AM de la capital Neuquina (LU5) y en el diario Sur Argentino, y fue contratado por Remus Tetu, durante el tiempo que duró su intervención, para llevar a cabo proyectos "de trabajos públicos de esta universidad". ${ }^{29} \mathrm{Y}$ si interesa destacar la persona de Guglielminetti, es justamente porque durante las acciones represivas que protagonizaría la PFA en este período y especialmente durante los primeros meses del gobierno dictatorial, él sería el nexo permanente entre el Ejército y la Delegación, ingresando y moviéndose en esta dependencia como si fuera una máxima autoridad de la institución, dirigiendo interrogatorios y llevando a cabo personalmente las sesiones de torturas realizadas en ese lugar. ${ }^{30}$ Dato que puede abonar a la reflexión en torno a la tensión entre autonomía y sistematicidad del proceso represivo, como veremos más adelante.

Otra muestra de la conexión entre la PFA, el Ejército y la Universidad Nacional del Comahue en el entramado represivo previo al golpe de Estado es el ingreso a esta casa de estudios de una decena de agentes de ambas fuerzas como estudiantes de distintas carreras, con el claro objetivo de realizar tareas de inteligencia. Todos ellos en 1975, plena gestión de Remus Tetu en la UNCo, y sin continuar en sus carreras más allá de ese año. Lo interesante del caso es que la inscripción a esas carreras se realizó de manera formal, por administración, y cada uno contaba con un legajo con sus nombres reales, sin ocultar su lugar de trabajo (sea en la Policía Federal, el Comando de Brigada o incluso uno de ellos como "Instructor en la Escuela de Policía"), y detallando como domicilios particulares las direcciones postales de las fuerzas a las que pertenecían. ${ }^{31}$ En este sentido, y teniendo en cuenta los testimonios que dan cuenta de cómo la universidad "estaba infiltrada" y que "había servicios por todos lados", no resulta forzado sostener que la función de estos "alumnos" pudo haber estado especialmente vinculada a la recolección de información y todo tipo de tareas de inteligencia, en pos de la persecución de estudiantes y trabajadores/as de esa casa de estudios.

\footnotetext{
${ }^{28}$ Fundamentos de la Sentencia del Tribunal Oral Federal en lo Criminal de Neuquén № 20/12 correspondiente a la "Causa Luera", 28/12/012, fs. 258/259 (en adelante, "Sentencia Causa Luera") ${ }^{29}$ Resolución UNCo "I" 0307/75

${ }^{30}$ Para un detalle más preciso de la participación de Guglielminetti en la represión, ver RFEJ 2011; Sentencia Causa Luera.

${ }^{31}$ Legajos de la UNCo reservados en Causa Reinhold; RFEJ 201, fs. 56
} 


\section{"Autonomía y sistematicidad del dispositivo represor. La Policía Federal en Neuquén (1975-1978)"}

\section{Autonomía y articulación, primera parte: Inteligencia}

El párrafo anterior nos lleva de alguna manera a caracterizar las tareas de inteligencia que desarrolló la Policía Federal en la Delegación neuquina, las cuales adquirieron un relativo grado de autonomía respecto del Ejército, más precisamente del D2 (o la División 2) del Comando de Brigada de Infantería de Montaña VI (quien tuvo a su cargo el desarrollo del plan represivo de la Subzona 52), así como del Destacamento de Inteligencia 182 que operaba en la misma manzana que el Comando y dependía directamente del Batallón 601 en Buenos Aires. ${ }^{32}$ Es preciso no olvidar que de acuerdo a los reglamentos y directivas militares, la labor de inteligencia era considerada una actividad prioritaria y precedente al resto de las operaciones a desarrollar en la "lucha antisubversiva", e implicaba una multiplicidad de tareas que incluían la obtención de todo tipo de información relativa al enemigo, sus acciones, organización interna y movimientos, y por lo cual era considerada de fundamental importancia ya que sin ella no se podían llevar a adelante ninguna de las operaciones previstas. ${ }^{33}$ Y más allá de la centralidad del despliegue represivo que tuvo el Ejército en esta región, la relativa autonomía que en ciertos aspectos tuvo la PFA -como al momento de obtener información y actuar en función de ella- resulta un dato que complejiza el cuadro y se vuelve necesario atender. En este sentido, la documentación disponible hasta el momento ${ }^{34}$ nos permite asegurar que ya en 1975 la Delegación neuquina de la PFA comenzó a realizar tareas de inteligencia de manera sistemática en vistas a combatir la "subversión". Y lo hacía en un doble plano fuertemente articulados entre sí: de manera relativamente autónoma mediante la acción de su propio personal -tanto en la producción de información como en su posterior análisis-, y al mismo tiempo coordinando el análisis de esa información con el de otras agencias estatales y paraestatales.

Así, además de lo señalado respecto de la vinculación de esta fuerza con la Universidad Nacional del Comahue desde comienzos de ese año -a través de Guglielminetti y de los "alumnos" infiltrados-, ambas instituciones participaban activamente en la denominada "comunidad informativa", en reuniones más o menos periódicas en las que representantes de todas las fuerzas de seguridad (Ejército, Gendarmería, policías provinciales de Río Negro y Neuquén, Policía Federal y SIDE)

\footnotetext{
${ }^{32}$ Para más detalle de la organización del Comando en la Subzona 52 ver Pablo Scatizza. Un Comahue violento... Op. Cit.

${ }^{33}$ Ver Directiva del Comandante General del Ejército Nro 404/75 “Lucha contra la Subversión” (1975); Directiva del Consejo de Defensa N 1/75 "Lucha contra la subversión” (1975); Reglamento RC-16-5 "La Unidad de Inteligencia" (1973)

34 Más allá de las fuentes mencionadas sobre el final del primer apartado, con las cuales hemos avanzado hasta aquí, tenemos conocimiento que en el Juzgado Federal N² de Neuquén -donde está radicada la Causa Reinhold ya aludida- cuenta con más documentación sobre este tema, así como expedientes relacionados con determinadas actuaciones de la Policía Federal previas al golpe de Estado. En este sentido, hemos solicitado a dicho juzgado la autorización para acceder a dichos expedientes, que al momento del cierre de la edición de este escrito (enero de 2018) no ha sido posible concretar.
} 


\section{Pablo Scatizza}

intercambiaban la información obtenida. ${ }^{35}$ Quién asistía a estas reuniones en representación de la PFA era el propio jefe de la Delegación, el comisario González, generalmente en compañía de algunos agentes a su cargo, entre ellos quienes trabajaban en la denominada "Oficina Técnica”, el área dentro de la Delegación que concentraba las tareas de inteligencia. ${ }^{36}$ En este último lugar, precisamente, los agentes -que vestían de civil y participaban también de los operativos represivos, como veremos a continuación- recibían y producían todo tipo de documentación de personas potencialmente sospechosas, y confeccionaban fichas con información relacionada con temas gremiales, socioeconómicos, estudiantiles y políticos. De esas fichas se realizaban cinco copias, tres de las cuales iban a Buenos Aires presuntamente a Coordinación Federal- y dos quedaban en la Delegación, una de las cuales era luego utilizada al momento del operativo relacionado con la persona relacionada con ella. ${ }^{37}$ La PFA contaba además con un grupo especial de tareas autodenominado "halcones", conformado por hombres que vestían de civil y ocupaban la Delegación generalmente de noche, que se encargaban de llevar, traer y procesar información, así participar de algunos operativos y sesiones de torturas. ${ }^{38}$

Esta vinculación de la PFA con otros actores del dispositivo represor que comienza a evidenciarse un año antes del golpe y se consolida luego del mismo, se completa con dos elementos para destacar, también en materia de inteligencia. Por un lado, con la participación activa de colaboradores civiles que concurrían asiduamente a la Delegación a brindar información relacionada con personas perseguidas por el régimen y, por otro, a partir de la particular y estrecha relación personal e institucional entre esta fuerza y algunos de sus agentes con el Ejército. Respecto a lo primero, si bien es sabido que las prácticas de delación formaron parte del entramado represivo, lo que por ahora es posible afirmar en este espacio -a partir de la documentación con la que contamos- es que hubo al menos dos sujetos activamente involucrados con esta dependencia policial en materia de aporte de información relacionada con la "lucha contra la subversión". Uno de ellos un comerciante repostero que vivía a la vuelta de manzana de la Delegación, y el otro un personaje que ha sido identificado por varios testigos de la Causa Reinhold llamado Alfonso Rodríguez, que no sólo compartió residencia con el comisario González, sino que trabajó un tiempo como supernumerario en la Delegación, durante el período en cuestión; además de haber trabajado en la Legislatura neuquina antes del golpe y en tareas propias de inteligencia y de persecución de estudiantes universitarios. ${ }^{39} \mathrm{El}$ segundo elemento a señalar también es sugerente, dado que permite analizar desde otra perspectiva -o bien con nuevos datos- el grado

\footnotetext{
${ }^{35}$ Los numerosos testimonios -como parte de la Causa Reinhold, en su mayoría- que dan cuenta de su funcionamiento señalan diferentes lugares donde esta "comunidad" se reunía, y que no siempre eran coordinadas por las mismas personas -incluso hay quienes señalan que, en 1975, los encuentros eran coordinados por el interventor de la UNCo, Remus Tetu.

${ }^{36}$ RFEJ 2011, p.58

37 Ibídem.

${ }^{38}$ Testimonial de Miguel Ángel Henríquez en TOCF, 2008

${ }^{39}$ RFEJ 2011, p. 57
} 


\section{"Autonomía y sistematicidad del dispositivo represor. La Policía Federal en Neuquén (1975-1978)"}

de descentralización que hubo entre los distintos circuitos represivos respecto de sus respectivos Comandos, así como del Consejo de Seguridad y la Junta Militar. En este sentido, algunos testimonios en la Causa Reinhold señalan que el comisario González era frecuentemente visitado en su oficina por personal del Destacamento de Inteligencia 182 (que dependía del Batallón 601 de Informaciones, en Buenos Aires, y operaba junto al Comando de Brigada de Infantería de Montaña VI, en la capital neuquina) y que mantuvo todo el tiempo estrechos vínculos personales con el Ejército. Asimismo, parte del personal de la Delegación que desarrollaba las mencionadas actividades de inteligencia revestía también -y al mismo tiempo- como Personal Civil de Inteligencia o bien como oficial de enlace con el Ejército ${ }^{40}$, lo que permite inferir que ello favorecía el intercambio de información y el mantenimiento de las relaciones entre ambas fuerzas.

Es interesante ver, entonces, cómo se fue configurando desde un año antes del golpe de Estado el entramado represivo en torno a las tareas de inteligencia, y cómo en esa red la Delegación de la Policía Federal cumplió un rol superlativo, dada su articulación con otros componentes del dispositivo represor. Es cierto que la propia comunidad informativa establecía un vínculo concreto entre las distintas fuerzas policiales y de seguridad, pero lo que deja en evidencia la dinámica propia de la PFA es que paralelo al funcionamiento de esa comunidad, tanto la Delegación como institución así como ciertos integrantes de la misma (como el comisario González, o los agentes con doble dependencia), además de producir y analizar su "propio" material de inteligencia, mantenían contacto permanente tanto con otras fuerzas estatales (Comando, Destacamento de Inteligencia 182, Coordinación Federal) como paraestatales (vía Remus Tetu y su fuerza de choque/Triple A) en materia de intercambio de información y de organización de las acciones represivas, que sin dudas favorecieron la puesta en acto del plan de exterminio en toda la región.

\section{Autonomía y articulación, segunda parte: Operativos}

La manera en que se llevaron a cabo los operativos de detención y secuestros desde la Delegación de la Policía Federal aportan un elemento más para pensar el grado de autonomía relativa que mantuvo esta fuerza con relación al Ejército, así como la manera en que actuó como componente articulador dentro de la propia dinámica del dispositivo represor. Si bien -como ya fue mencionado- luego de los decretos de octubre de 1975 esta y todas las fuerzas policiales y de seguridad quedaron bajo el control operacional del Ejército, es importante destacar que dicho control no fue tan estricto como sí lo fue, por ejemplo, para con las policías provinciales o la Unidad № 9 del Servicio Penitenciario Federal (U9), donde fueron alojadas o trasladadas temporalmente la mayoría de los/as detenidos/as por motivos políticos.

40Ibídem. 


\section{Pablo Scatizza}

Como vimos en el apartado anterior, ya desde las tareas de inteligencia la PFA mantuvo un relativo grado de autonomía dentro del entramado represivo, que si bien se mantuvo al momento de llevar a cabo los operativos, tuvo al menos una característica que creo importante destacar. En este sentido, y dado el rol "natural" (histórico) de esta fuerza respecto de su intervención en delitos comunes de competencia federal, durante este período queda en evidencia la facultad que tuvo para articular distintos objetivos represivos, como los que se unificaron en la triada juventud, drogas y subversión.

Juventud, drogas y "subversión". Una triada contra la "seguridad nacional"

Detengámonos ahora a observar una práctica muy específica desarrollada en la Delegación de la Policía Federal, cuyo análisis nos va a permitir demostrar aún con más claridad el grado de autonomía, arbitrariedad y abuso de poder desplegado por esta fuerza durante el período analizado, en especial por los hombres que estuvieron a su cargo entre 1975 y 1977. Una práctica que, a la luz de algunas nuevas preguntas, podría llevarnos a modificar significativamente algunas hipótesis que teníamos, hasta el momento, respecto al accionar del dispositivo represor. Una práctica que se habría propuesto eliminar una triada que atentaba peligrosamente contra la "seguridad nacional", conformada por la juventud, las drogas y la subversión. ${ }^{41} \mathrm{Si}$ bien aún no ha sido posible acceder a una mayor cantidad de documentación que permitiera asegurar con mayor énfasis lo que sigue a continuación, los dos casos que referiré permiten redefinir aquella hipótesis respecto al accionar de la PFA en Neuquén. Nos referimos puntualmente a una forma de persecución y represión de la "subversión" desplegada desde la Delegación ya en dictadura (es posible que también durante el año anterior, pero aún no ha sido posible acceder a documentación que lo demuestre), basada en el armado de causas por infracción a la "ley de drogas" (Ley 20.771) para perseguir y reprimir "legalmente" a grupos de jóvenes que, en tanto podían tener vínculos con la droga, también podían (o no) tenerlos con organizaciones políticas, pero que de una forma u otra representaban un peligro para la "seguridad nacional" que debía ser combatido.

Se trata, concretamente, de dos causas judiciales diferentes, por infracción a la Ley 20.771, que involucraron a dos víctimas que forman parte de la causas por delitos de lesa humanidad, Roberto Saez y Jorge Onofri, cuyos casos fueron ventilados en el cuarto juicio oral realizado en el Tribunal Oral Federal en lo Criminal de Neuquén entre 2015 y 2016. ${ }^{42}$ Dado que por cuestiones de espacio no es posible detallar aquí cada uno de esos casos, baste decir por ahora que, en ambos hechos los nombrados fueron agregados a ciertas causas iniciadas en la Delegación de la PFA, luego de ser mencionados por personas que ya formaban parte de esos expedientes, durante las declaraciones indagatorias realizadas en esa institución por el propio

\footnotetext{
${ }^{41}$ Esta noción es tomada de Valeria Manzano. "The Creation of a Social Problem: Youth Cultures, Drugs, and Politics in Cold War Argentina". Hispanic American Historical Review, vol. 95, no. 1, 2015, a cuyo trabajo refiero a continuación.

${ }^{42}$ La decisión de aportar el nombre y apellido de estas personas estriba en que sus casos fueron ventilados en juicio oral y público, por lo cual no hay identidad que sea necesario preservar.
} 


\section{"Autonomía y sistematicidad del dispositivo represor. La Policía Federal en Neuquén (1975-1978)"}

comisario Jorge Ramón González y sus agentes. Menciones que los vinculaban al comercio y consumo de estupefacientes, por lo cual de manera inmediata se procedía a su detención. En ambos casos permanecieron detenidos en la Delegación durante varios días, tiempo en el que fueron maltratados física y psicológicamente, e interrogados bajo tortura. En esos interrogatorios, se les preguntaba por cuestiones vinculadas a las drogas y respecto a personas de la política y la militancia social.43 Mientras Onofri fue liberado finalmente una semana después de su detención, Saez fue involucrado en otra causa -por el propio comisario Gonzálezjusto el día que iba a ser liberado, vuelto a detener, y trasladado a la Compañía de Comunicaciones de Montaña № 6 donde permaneció detenido durante cuatro meses. En ese período, fue trasladado al Centro Clandestino de Detención (CCD) "La Escuelita", donde permaneció ocho días siendo sistemáticamente torturado e interrogado.

Es necesario señalar que en un primer momento -y así quedó expresado en otro escrito e, incluso, en el Requerimiento Fiscal de Elevación a Juicio de cuya elaboración formó parte quien suscribe ${ }^{44}$ - supusimos que ese modus operandi se trataba de la creación deliberada de causas judiciales por consumo o tenencia de estupefacientes en las que se involucraba de manera falsa a personas que buscaban detener e interrogar por motivos políticos, o bien mediante la incorporación de esas personas en causas -por infracción a la ley de drogas- ya existentes. Ello, teniendo en cuenta que por aquel entonces, debido a la vigencia de la Ley 2.372 (¡del año 1888!), los funcionarios policiales tenían la facultad de detener personas contra quienes hubiera "indicios vehementes o semiplena prueba de culpabilidad", a "conservar incomunicado al detenido", "a recibir a las personas detenidas declaración espontánea en la misma dependencia policial y a producir la prueba que juzgaren necesaria". ${ }^{45}$ De esta manera, creímos, la Policía Federal tenía en sus manos las herramientas necesarias para poder detener "legalmente" a las personas que buscaban por motivos políticos, endilgándoles el carácter de imputados en una prevención sumarial, retenerlas en el ámbito físico de la Delegación -de manera "legal"- y someterlas a interrogatorios para obtener de ellos información que sirviera a la "lucha antisubversiva" bajo aplicación de tormentos.

Si bien esa fue la hipótesis que mantuvimos hasta aquí, había una pregunta que no dejaba de inquietarnos y que la ponía en crisis nuestra teoría: si el objetivo era detener por motivos políticos a ciertas personas, ¿por qué inventar una causa judicial (que legalizara esa detención), si antes y después hubo detenciones ilegales

\footnotetext{
${ }^{43}$ En el caso de Saez se lo interrogó respecto al Obispo Jaime de Nevares y su militancia barrial (Saez había militado en el peronismo), y en el caso de Onofri, acerca de militantes y personas que ya habían estado detenidas -e incluso desaparecidas- vinculados al teatro, así como por la presunta conexión con el MIR chileno de, justamente, una mujer llamada Fresia, que él había conocido días antes de su detención y por la cual terminó siendo detenido. Cfr. RFEJ 2011.

${ }^{44}$ Entre 2008 y 2014 formé parte del equipo de Derechos Humanos de la Fiscalía Federal de Neuquén, con la tarea de armar el entramado histórico del período investigado en los juicios de lesa humanidad. El escrito aludido es el libro Un Comahue violento, ob. cit.

${ }^{45}$ Art. 184 del Código de Procedimientos en Materia Penal, Ley 2.372.
} 


\section{Pablo Scatizza}

y secuestros protagonizados por los mismos perpetradores, y no hay indicios que evidencien algún tipo de preocupación por esa situación de irregularidad?

Fueron los trabajos de Valeria Manzano ${ }^{46}$ en torno a la creación de un problema político-social por parte del Estado sobre las drogas y la juventud en los setentas los que nos ayudaron a construir nuevas preguntas, cuyas potenciales respuestas le aportaron un giro a la investigación. En su estudio, Manzano desarrolla una hipótesis más que sugerente al señalar que la sanción en 1974 de la Ley 20.771 fue una pieza más del andamiaje de legislación represiva promulgada ese año, junto a la promulgación de otras normas como con la ley de asociaciones profesionales, la reforma del código penal y la ley universitaria que limitaba la actividad política en las aulas. ${ }^{47}$ Para demostrarlo, la historiadora recorre el entramado discursivo y político que rodeó la génesis, sanción y aplicación de esta ley hasta su reemplazo por una nueva en 1989, destacando cómo el consumo y la tenencia de drogas fue convertido en un "problema" a comienzos de la década del '70, trasladando el foco de atención desde el tráfico y los traficantes hacia los consumidores -que por definición eran jóvenes-, para finalmente colocar el desde entonces "problema de las drogas" dentro de la esfera de la "seguridad nacional". ${ }^{8}$

Frente a este dato, una primera pregunta en torno a nuestro objeto de estudio surgió de manera inmediata, casi de manera natural: la detención en el marco de causas por drogas de estas personas que luego fueron interrogadas sobre militantes y referentes de la política regional, ¿fue sólo una manera de "blanquear" un secuestro por motivos políticos, o había algo más que no estábamos viendo? ¿Este tipo de detenciones formaban parte de su rutina de funcionamiento, o estaban relacionadas -y hasta qué punto- con la "lucha contra la subversión”? ¿Era posible que la propia Policía Federal neuquina haya actuado pensando en esa triada drogas-jóvenessubversión? Pero si fue así, ¿por qué y a partir de qué indicios interrogaron solo a uno de los imputados en cada una de esas causas por personajes de la política? ¿Y si no fue sólo a ellos a quienes se interrogó bajo apremios sobre cuestiones políticas, sino que sólo nos enteramos de esos dos hechos porque fueron los casos denunciados a la justicia luego de la dictadura y que a la postre integraron los juicios por delitos de lesa humanidad? ¿Es posible suponer que el resto de los/as imputados/as en esas causas por drogas también hayan sido interrogados respecto

\footnotetext{
${ }^{46}$ Específicamente Valeria Manzano. "The Creation of a Social Problem: Youth Cultures, Drugs, and Politics in Cold War Argentina". Op. Cit., y una ponencia de su autoría (inédita) en el marco de las III Jornadas de la Red de Estudios sobre Represión y Violencia Política realizada en La Plata (FaHCE) en abril de 2017.

${ }^{47}$ Para mayor detalle de esta legislación represiva ver Marina Franco. Un enemigo para la nación...Op.Cit.

${ }^{48}$ Manzano destaca cómo el Ministro de Bienestar Social del gobierno de facto de Alejandro Lanusse, Francisco Manrique, ya en 1972 y en el marco de la creación de la Comisión Nacional de Toxicomanía y Narcóticos (CONATON), sostuvo que "el consumo de drogas corroe la moral de los jóvenes y los mueve a seguir a grupos que tratan de subvertir el orden social", y que por ello "la droga" era un problema "de seguridad y de salud". Asimismo, su sucesor en ese cargo, José López Rega, plantearía algo similar al año siguiente al subrayar que "nuestro compromiso mutuo es luchar contra el mal de las drogas y de la subversión" ("The Creation of a Social Problem...Op. Cit, p. 53)
} 


\section{“Autonomía y sistematicidad del dispositivo represor. La Policía Federal en Neuquén (1975-1978)"}

de cuestiones políticas, pero que no lo supiéramos dado que no denunciaron sus casos en el marco de los juicios de lesa humanidad?

Al mirar con más detalle y bajo estas nuevas preguntas los expedientes que involucraron a estas dos personas, y a partir de las declaraciones realizadas por las personas imputadas en esas causas ya en sede judicial ${ }^{49}$ (luego de la instrucción realizada en la Delegación) vemos que la práctica de obtener confesiones -algo sustancial en el sistema judicial inquisitorial en boga por entonces- bajo torturas se repite en la mayoría de los involucrados en dichas expedientes, así como la exigencia de firmar supuestas declaraciones sin leer. Esto fue denunciado por varios/as de los imputados/as en esas causas cuando el expediente fue elevado a sede judicial. Jorge Onofri declaró incluso que escuchó cómo torturaban y aparentemente violaban a una de las mujeres imputadas en la misma causa que él, por la cual, incluso, le preguntaron qué conexiones tenía con el MIR chileno dado que ella provenía de ese país. ${ }^{50} \mathrm{Y}$ en este sentido, si seguimos esta nueva hipótesis, no es demasiado aventurado suponer que en la lógica de los perpetradores, si era "chilena y drogadicta" bien podía pertenecer a esa organización revolucionaria trasandina (de la cual ya se sabía que tenía vínculos con el PRT-ERP). ${ }^{51}$ Es cierto que sólo sabemos que sólo a Onofri y a Sáez los interrogaron por sus conexiones o conocimiento de personas vinculadas a la política regional. Pero eso lo sabemos porque sus casos fueron los únicos, de sus respectivos expedientes, que fueron conocidos luego de la dictadura, y cuyo testimonio ha sido posible obtener en la actualidad. ¿Cómo es posible asegurar que al resto de los imputados en esas causas por drogas no los interrogaron con preguntas similares, especialmente cuando lo hicieron bajo la aplicación de tormentos?

Otro dato que quisiéramos dejar mencionado acá es el grado de "represión moral" que se desprende de estos expedientes, lo cual de alguna manera, junto con lo anterior, nos obliga a reformular nuestras concepciones sobre la represión estatal en este período. ${ }^{52}$ En tal sentido, es recurrente, por ejemplo, la manera en que los policías indagan en sus interrogatorios en torno a la homosexualidad de los imputados, hurgando en detalles de las prácticas sexuales cuya descripción ocupa numerosas líneas en cada uno de los expedientes. Tal es así, que en una de las causas citadas el Juez le pide un Informe Pericial al médico forense para que realice sobre uno de los imputados un análisis de "su estado físico anal", dado que "resulta

\footnotetext{
${ }^{49}$ Queda para el momento de completar esta investigación estudiar más en detalle la participación del Juez Federal en estos expedientes, especialmente el que tuvo como protagonista al entonces juez Pedro Duarte, pieza clave del dispositivo represor.

${ }^{50}$ Ver Testimonial de Jorge Onofri en TOFC, 2016

${ }^{51}$ Testimonio de José Luis Sexton en Expte 303 Cámara Federal de Bahía Blanca (agregado a la Causa Reinhold)

${ }^{52}$ En este sentido también están avanzando, cabe destacar, Ana Sanchez Troillet a partir de sus estudios sobre jóvenes rockeros, policías y cultura urbana; Fedra López Perea en torno a la represión estatal contra sexualidades no heteronormativas; Valeria Snitcofsky y sus avances sobre la erradicación de villas urbanas; y Diana Lenton en torno a las formas de represión sobre los pueblos originarios.
} 


\section{Pablo Scatizza}

damnificada la salud pública".53 Informe que el médico concluye asegurando que "el citado podría tratarse con bastantes seguridades (sic) de un homosexual pasivo, lo cual se vería reforzado mediante un estudio psiquiátrico u otras pruebas". ¿Hasta qué punto este tipo de prácticas estaba relacionada con la "lucha antisubversiva"? ¿O formaba parte de la rutina cotidiana -en términos represivos- del funcionamiento de la Policía Federal? 0, cruzando ambos interrogantes, ¿en qué manera ciertas prácticas rutinarias de esta fuerza -y también de otras, por supuesto- fueron incorporadas a la lucha contra la "subversión"?

\section{La PFA como centro de detención clandestina}

Además de la modalidad operativa descripta en el apartado anterior, la Delegación de la Policía Federal actuó activa y protagónicamente en una gran cantidad de raids represivos, tanto el año anterior al golpe como en los subsiguientes. Actuación que se efectuó tanto a partir de la integración de Grupos de Tarea, vestidos de civil o bien disfrazados en aquellos casos en que se trató de operativos de secuestro, como conformando Comisiones de Detención, así fuera acompañando a oficiales del Ejército o encabezando y dirigiendo los operativos no encubiertos. ${ }^{54}$ En no pocas de las acciones represivas encabezadas por la PFA, quien comandó los operativos fue el ya mencionado Raúl Guglielminetti, quien, como vimos más arriba, tuvo la cualidad de articular la dinámica represiva entre las distintas fuerzas, especialmente entre el Ejército (en su rol de PCI) y la Policía Federal.55 Articulación que incluyó, por ejemplo, el traslado personal de detenidos/as desde y hacia la Delegación, la U9 del Servicio Penitenciario Federal y el CCD "La Escuelita”.

Por otro lado, una de las principales características que adoptó la Delegación de la Policía Federal ya durante la dictadura fue la de funcionar como Centro Clandestino de Detención. Aunque tal como lo hemos precisado en otros trabajos, sería más preciso describirlos como centros de detención clandestina, y destacar la condición de clandestinidad en la forma en que se mantenían allí a los detenidos, más que propiamente al lugar que sirvió como tal. ${ }^{56} \mathrm{Y}$ ello, dado que su principal característica frente a los CCD como "La Escuelita”, que funcionó en los fondos del Batallón de Ingeniero de Montaña VI, es que se trató de lugares públicos,

\footnotetext{
${ }^{53}$ Expte. $361-\mathrm{F}^{\circ} 888$, fs. 47 vta. El subrayado es nuestro.

${ }^{54}$ Las Comisiones de Detención estaban conformadas por personal del Ejército y de las policías provinciales y Federal. Actuaban vestidos de civil o con uniforme y generalmente se identificaban al practicar las detenciones. Los Grupos de Tarea, en cambio, operaban de manera clandestina y mucho más violenta, en vehículos civiles, sin identificación o con identificaciones falsas, y sus integrantes actuaban vestidos de civil, con el rostro oculto (utilizando capuchas, bufandas, pañuelos) o directamente disfrazados.

55Por cuestiones de espacio no es posible detallar aquí estos operativos. Para más información, ver RFEJ 2011; Pablo Scatizza. Un Comahue violento... Op. Cit.

${ }^{56}$ Para más detalle sobre estos espacios de reclusión y su caracterización ver Pablo Scatizza. "Centros clandestinos de detención en el Comahue. Una reflexión conceptual". Contenciosa, Año I, Nro. 2, primer semestre 2014 [En línea en http://www.contenciosa.org/Sitio/VerArticulo.aspx?i=18 ; Un Comahue violento... op. cit. pp. 185-213
} 


\section{“Autonomía y sistematicidad del dispositivo represor. La Policía Federal en Neuquén (1975-1978)"}

identificados claramente por la comunidad neuquina. En este sentido, las personas detenidas -legal e ilegalmente- eran ingresadas por la puerta principal, esposadas y con la cara descubierta, y la rutina diaria parecía no alterarse a pesar de las acciones clandestinas que allí se llevaban a cabo. El movimiento normal del personal de la institución no se interrumpía, quienes continuaban con sus tareas administrativas habituales, incluso atendiendo al público. Y, mientras ello sucedía, en las oficinas ubicadas al fondo eran retenidas en condiciones inhumanas las personas detenidas allí ingresadas, así como interrogadas y torturadas. A lo largo del período analizado -en especial entre 1975 y 1977- ha sido posible identificar a diecisiete víctimas que fueron trasladadas y mantenidas clandestinamente a la Delegación de la PFA, aunque ese número podría ser mucho mayor. ${ }^{57}$ Personas que fueron siempre retenidas de manera clandestina, incomunicadas, en su mayoría sometidas a malos tratos y torturas, e interrogadas por sus vinculaciones políticas y su militancia partidaria.

Si bien hay registros de la utilización de esta dependencia como CCD hasta entrado 1977, la mayor intensidad de su protagonismo como lugar de reclusión clandestina la tuvo en los primeros meses luego del Golpe, dado que "La Escuelita", el principal espacio de concentración que funcionó durante la dictadura y que estuvo bajo control operacional y funcional del Ejército, comenzó a operar a mediados de junio de 1976 al momento de ejecutarse un operativo para eliminar al PRT-ERP de la región.

\section{Entre la autonomía y la sistematicidad}

Es hora de volver a la pintura que abre este escrito y pensar si el juego de escalas tuvo el efecto deseado. El lienzo es el mismo, pero ¿lo es también la significación que ahora podemos otorgarle? ¿Cuál es el grado relativo de luz que la pincelada que define a la Policía Federal de Neuquén -y su accionar- le aportan al cuadro de la represión estatal durante la última dictadura militar y sus años previos? ¿Es sólo un punto de color claramente definido, o el acercamiento mediante un lente de mayor aumento nos muestra -o nos permite significar- una serie de matices que lo complejizan?

El rol que le cupo a la Policía Federal en el despliegue represivo en todo el territorio nacional ha sido demostrado profusamente en los últimos años, y a lo largo de este escrito hemos referido a parte de la basta bibliografía que da cuenta de ello. En menor medida, también ha comenzado a investigarse su accionar en la Patagonia Norte, y ya ese cambio de escala espacial ha permitido observar algunas tonalidades

\footnotetext{
${ }^{57}$ En una entrevista personal que mantuvimos con Orlando Balbo, una de las primeras personas que fue secuestrada el propio 24 de marzo, trasladada y torturada en la PFA, recordó que cuando fue llevado a ese lugar por sus secuestradores, "no entraba un alma más en la Delegación", ya que estaba llena de jóvenes estudiantes de una residencia universitaria que había en el barrio La Sirena. Allí habían ido las fuerzas de seguridad "en busca de un grupo de militantes y, ante la duda, se llevaron a todos los residentes". De acuerdo a lo que pudo averiguar después, la mayoría de esos jóvenes fue fichado y puestos en libertad en los días siguientes.
} 


\section{Pablo Scatizza}

diferentes, a las cuales este trabajo que aquí concluye pretende abonar aumentando un poco más la lente de observación, tratando de aportar mayor complejidad a la noción de sistematicidad del proceso represivo, especialmente en relación/tensión con los grados de autonomía relativa que tuvieron determinadas fuerzas que actuaron en él.

Como vimos, la Delegación de la Policía Federal de Neuquén tuvo un rol protagónico en el despliegue del dispositivo represor desde un año antes del golpe de Estado, que se prolongó durante los meses de mayor intensidad de la violencia estatal de la dictadura militar. En ese período, tanto en materia de inteligencia como de detenciones, secuestros, retención de detenidos, interrogatorios y otras prácticas represivas, su actuación se desarrolló en dos planos articulados entre sí: de manera relativamente autónoma mediante el accionar de su propio personal en determinados casos (tanto en la producción de información y su posterior análisis como en la posterior ejecución de las operaciones), y al mismo tiempo coordinando el análisis de su información y ciertos operativos con el de otras agencias estatales y paraestatales. Esto le permitió a la PFA desarrollar sus actividades con un relativo grado de autonomía respecto del Poder Judicial durante el período constitucional ${ }^{58}$, así como de las Fuerzas Armadas luego de octubre del ' 75 cuando los decretos de aniquilamiento ordenaron que bajo ellas quedaría el control operacional en materia de "lucha antisubversiva".

¿Implica esta autonomía la descentralización de los objetivos primarios que persiguieron las Fuerzas Armadas, concretados con su asalto al poder estatal? ¿Niega el accionar relativamente independiente de la PFA la organización y puesta en acto de un plan sistemático de exterminio y terror en todo el país por parte de las Fuerzas Armadas? De ninguna manera. Si algo muestra y confirma la manera en que la PFA intervino en el desarrollo de la represión en la Norpatagonia, justamente, es la potencia que tuvo dicha planificación. Potencia que habilitó la existencia y convivencia de diferentes grados de autonomía y acción por parte de las distintas fuerzas y agencias que formaron parte del dispositivo represor que se desplegó en todo este período. En este sentido, si algo deja en claro y expresa el análisis aquí presentado del accionar de la Policía Federal en Neuquén, es otra muestra más de la estrategia elaborada por las Fuerzas Armadas en los años previos al golpe de Estado, para llevar a cabo su "guerra contrainsurgente", que se ejecutó mediante el accionar represivo descentralizado a través de grupos militares, policiales y civiles, los cuales actuaban bajo una dirección centralizada (militar) aunque manteniendo un relativo grado de autonomía. ${ }^{59}$

\footnotetext{
${ }^{58} \mathrm{Si}$ bien definimos al comienzo que nos interesaba indagar en la relativa autonomía de la PFA en la planificación y ejecución de las acciones represivas respecto de las Fuerzas Armadas, no está demás señalar esta relativa independencia que también tuvo en su accionar en relación con el Poder Judicial, tal como vimos en el cuarto apartado de este escrito.

${ }^{59}$ Esteban Pontoriero. "En torno a los orígenes del terror de Estado en la Argentina de la década de los setenta. Cuándo, cómo y por qué los militares decidieron el exterminio clandestino". Papeles de Trabajo, 10(17), 2016, pp. 30-50.
} 


\section{“Autonomía y sistematicidad del dispositivo represor. La Policía Federal en Neuquén (1975-1978)"}

Si bien la conducción del accionar represivo en la Subzona 52 estuvo ubicada en el Comando de la VI Brigada de Infantería de Montaña, y la Policía Federal actuó bajo control operativo de esa fuerza a partir de sancionados los "decretos de aniquilamiento" de octubre de 1975, hemos podido ver cómo esta fuerza manejó un grado de autonomía no menor al momento de llevar a cabo sus acciones. Pero aun así, persiguiendo siempre el claro y preciso objetivo de "combatir y aniquilar a la subversión".

\section{Bibliografía citada}

Ana Belén Zapata. "Andamios de experiencias". Conflictividad obrera, vigilancia y represión en Argentina. Bahía Blanca, 1966-1976. Tesis doctoral, Universidad Nacional de la Plata, 2014. mimeo

Ariel Eidelman. El desarrollo de los aparatos represivos del Estado argentino durante la "Revolución Argentina", 1966-1973, Tesis de Doctorado, UBA, 2010. Disponible en http://repositorio.filo.uba.ar/handle/filodigital/1412

Diego Kenis. “Quién fue Remus Tetu?”, Agencia Paco Urondo, 3/10/2015. Disponible en http://www.agenciapacourondo.com.ar/ddhh/quien-fue-remustetu.

Eduardo Luis Duhalde. El Estado Terrorista Argentino. Quince años después. Buenos Aires, Eudeba, 1999

Esteban Pontoriero. "En torno a los orígenes del terror de Estado en la Argentina de la década de los setenta. Cuándo, cómo y por qué los militares decidieron el exterminio clandestino". Papeles de Trabajo, 10(17), 2016, pp. 30-50

Gabriela Águila, Santiago Garaño y Pablo Scatizza (coords.). Represión estatal y violencia paraestatal en la historia reciente argentina: nuevos abordajes a 40 años del golpe de Estado. La Plata, Ediciones FaHCE, Universidad Nacional de La Plata, 2016. Disponible en: http://www.libros.fahce.unlp.edu.ar/index.php/libros/catalog/book/63

Gabriela Águila. "La represión en la historia reciente argentina: fases, dispositivos y dinámicas regionales". En Gabriela Águila y Luciano Alonso (coord.). Procesos represivos y actitudes sociales. Entre la España franquista y las dictaduras del Cono Sur. Buenos Aires, Prometeo, 2013, pp.97-121

Gabriela Águila. Dictadura, represión y sociedad en Rosario, 1976/1983. Un estudio sobre la represión y los comportamientos y actitudes sociales en dictadura. Buenos Aires, Prometeo, 2008

Hernán Merele, "La "depuración ideológica” del peronismo en el Partido de General Sarmiento (Buenos Aires). Una aproximación a partir del caso de Antonio "Tito" 


\section{Pablo Scatizza}

Deleroni", Ponencia presentada en las Primeras Jornadas de Historia Reciente del Conurbano Bonaerense Norte y Noroeste, UNGS, Buenos Aires, agosto de 2013

Horacio Paino. Historia de la Triple A. Montevideo, Editorial Platense, 1984

Humberto Zambón. La misión Tetu en el Comahue. Neuquén, Educo, 2008

Ignacio González Janzen. La Triple A. Buenos Aires, Contrapunto, 1987

Jacques Revel (director). Juego de escalas. Experiencias de micronalálisis. Buenos Aires, UNSAM Edita, 2015 [1996]

Juan Quintar, Beatriz Gentile, Susana Debattista y Carla Bertello. "La Universidad Nacional del Comahue en los años 70: de la rebelión creativa a la reacción autoritaria". En AA.VV., Universidad Nacional del Comahue 1972-1997. Una historia de 25 años. Neuquén, Educo, 1998

Marcelo Larraquy. Lopez Rega. El peronismo y la Triple A. Buenos Aires, Punto de Lectura, 2007

Marcos Novaro y Vicente Palermo. La dictadura militar (1976/1983). Del golpe de estado a la restauración democrática. Buenos Aires, Paidós, 2006

Marina Franco. Un enemigo para la nación. Orden interno, violencia y "subversión", 1973-1976. Buenos Aires, FCE, 2012

Martín Andersen. Dossier Secreto. El mito de la guerra sucia. Buenos Aires, Planeta, 1993

Martín Andersen. La policía. Pasado, presente y futuro. Buenos Aires, Sudamericana, 2002

Michel Foucault. "El juego de Michel Foucault", en Saber y Verdad. Madrid, Las Ediciones de la Piqueta, 1984

Pablo Scatizza. "Centros clandestinos de detención en el Comahue. Una reflexión conceptual". Contenciosa, Año I, Nro. 2, primer semestre 2014 [En línea en http://www.contenciosa.org/Sitio/VerArticulo.aspx?i=18

Pablo Scatizza. Un Comahue violento. Dictadura, represión y juicios en la Norpatagonia argentina. Buenos Aires, Prometeo, 2016

Pablo Scatizza. "Represión 'antisubversiva' en la Norpatagonia. Estrategias estatales y paraestatales de persecución política en Neuquén y Río Negro (1973-1976)”. Papeles de Trabajo, 10 (17), pp. 52-72

Peter Burke (ed.). Formas de hacer historia. Madrid, Alianza, 1994 
“Autonomía y sistematicidad del dispositivo represor. La Policía Federal en Neuquén (1975-1978)"

Pilar Calveiro. Poder y desaparición. Los campos de concentración en Argentina. Buenos Aires, Colihue, 2006

Prudencio García. El drama de la autonomía militar. Argentina bajo las juntas militares. Madrid, Alianza, 1995

Sergio Buffano."Perón y la Triple A". Lucha Armada en la Argentina Año $1-N^{\circ} 3$, Buenos Aires, 2005

Valeria Manzano. "The Creation of a Social Problem: Youth Cultures, Drugs, and Politics in Cold War Argentina". Hispanic American Historical Review, vol. 95, no. 1, 2015

Recibido: 08/10/2017

Evaluado: $15 / 11 / 2017$

Versión Final: 27/12/2017 